\title{
Results of an Enhanced Recovery after Surgery Protocol for Upper Gastrointestinal Surgery at a Super-Tertiary Referral Hospital in Thailand
}

\author{
Thammawat Parakonthun ${ }^{1,2}$, Thikhamporn Tawantanakorn ${ }^{1}$, Jirawat Swangsri, ${ }^{1,2^{*}}$, \\ Tharathorn Suwatthanarak ${ }^{1}$, Nicha Srisuworanan ${ }^{1}$, Voraboot Taweerutchana ${ }^{1}$, \\ Chainarong Phalanusitthepa ${ }^{1}$, Atthaphorn Trakarnsanga ${ }^{1}$, Thawatchai Akaraviputh ${ }^{1}$, \\ Asada Methasate ${ }^{1,2}$, Vitoon Chinswangwatanakul ${ }^{1}$
}

\author{
*Corresponding author: \\ Jirawat Swangsri, MD, PhD, \\ Assistant Professor of Surgery \\ Minimally Invasive Surgery Unit \\ Division of General Surgery \\ Department of Surgery \\ Faculty of Medicine Siriraj Hospital \\ Mahidol University \\ 2 Wang Lang Road, Bangkoknoi \\ Bangkok, 10700, Thailand \\ Tel: (+66) 2-419-8005 \\ Fax: (+66) 2-412-1370 \\ E-mail: oatjiro@yahoo.com
}

ERAS: Enhanced recovery after surgery; UGI: upper gastrointestinal; EGJ: esophagogastric junction; PONV: Postoperative nausea and vomiting;

BMI: body mass index;

BW: Body weight;

\section{Abbreviations:}

\author{
${ }^{1}$ Minimally Invasive Surgery Unit, Division of General Surgery, Department of Surgery, \\ Faculty of Medicine Siriraj Hospital, Mahidol University, Bangkok, Thailand \\ ${ }^{2}$ Siriraj Upper Gastrointestinal Cancer Center, Faculty of Medicine Siriraj Hospital, \\ Mahidol University, Bangkok, Thailand
}

\section{ABSTRACT}

Background: Enhanced recovery after surgery (ERAS) protocol has been shown to reduce recovery period and postoperative complications. This prospective study aimed to evaluate the short-term outcomes of ERAS in patients who underwent upper gastrointestinal (UGI) surgery.

Methods: Patients who underwent surgery for benign or malignant diseases of the esophagus, esophagogastric junction, or stomach during October 2018-December 2019 were included. Outcomes compared between ERAS and conventional care (CC) included length of hospital stay (LOS), postoperative complications, mortality, recovery, and hospitalization cost.

Results: Eighty-eight (32 ERAS, $56 \mathrm{CC}$ ) patients were included. ERAS had shorter LOS (5.5 vs. 12.5 days, $p<0.001$ ), earlier time to toleration of oral soft diet ( 3 vs. 8 days, $p<0.001$ ), faster time to first defecation (71.6 vs. 114.5 hours, $p<0.001$ ), early ambulation (43.8 vs. 84.6 hours, $p=0.010)$, and reduced hospitalization cost (197,140 vs. 256,315 Thai baht, $p<0.032$ ). C-reactive protein level on postoperative day 1 was significantly lower in ERAS patients (61.3 vs. $87.9 \mathrm{mg} / \mathrm{L}, p=0.027$ ). BMl recovery occurred within 3 and 6 months in ERAS and CC, respectively. There was no significant difference in surgery-related or nonsurgery-related complications between groups. No mortality was observed.

Conclusions: In a UGI surgical setting, ERAS accelerated recovery, reduced LOS, and lowered hospitalization cost.

Key words: Enhanced Recovery after Surgery (ERAS), upper gastrointestinal surgery, short-term outcomes, complications, hospitalization cost

\section{INTRODUCTION}

Accepted: 10.07 .2020

Copyright () Celsius Publishing House www.sgo-iasgo.com
Upper gastrointestinal (UGI) surgery, including esophageal and gastric surgery, are high-risk procedures that are associated with a high rate of perioperative complications (1). Enhanced Recovery after Surgery (ERAS), also 
known as fast track surgery, was initiated by Henrik Kehlet in the 1990s (2). ERAS is a multidisciplinary approach that aims to promote early postoperative recovery, reduced surgical stress response and organ dysfunction, decreased postoperative complications, reduced length of hospital stay and hospitalization cost, and improved long-term survival (3-5). ERAS has been successfully applied in many fields of surgery, including gastric and esophageal surgery (6-8). Although the safety and efficacy of the ERAS protocol in UGI surgery has been reported in numerous studies, implementation of the ERAS protocol is a challenge, and this has led to slow adoption of ERAS in some settings (9). Moreover, successful implementation requires protocol compliance by all members of the multidisciplinary team.

ERAS for UGI surgery is becoming the new standard of care in Thailand. The intensive ERAS protocol reported in this study was developed by UGI surgeons and the other members of the multidisciplinary team at Siriraj Hospital, which is Thailand's largest national tertiary referral center. We developed a perioperative protocol for patients undergoing UGI surgery that is based on evidence-based literature $(10,11)$. The purpose of this prospective study was to assess shortterm outcomes compared between ERAS protocol and conventional care among patients who underwent UGI surgery.

\section{MATERIALS AND METHODS}

\section{Study design and patient selection}

This single-center prospective cohort study was designed to evaluate the short-term postoperative outcomes of patients who received the ERAS protocol among patients who underwent UGI surgery. All patients diagnosed with benign or malignant diseases of the esophagus, esophagogastric junction (EGJ), or stomach who underwent elective surgery during 1 October 2018 to 31 December 2019 at the Faculty of Medicine Siriraj Hospital, Mahidol University, Bangkok, Thailand were recruited. The eligibility criteria were age 18 years or older and American Society of Anesthesiologists (ASA) score of 4 or less. Patient study group assignment was made by consensus decision between the patient and his/her physician. All patients received standardized surgery and routine surveillance. Conventional perioperative care was defined as the normal perioperative management given and ordered by individual surgeons based on their knowledge and experience.

\section{Enhanced Recovery after Surgery (ERAS) protocol}

Patients and their care givers received preoperative counseling and education regarding the treatment plan and preoperative preparation. Smoking and alcohol drinking were prohibited at least 2 to 4 weeks prior to surgery (12). Breathing exercise and chest physical therapy were encouraged preoperatively (10). An anesthesiologist was consulted to evaluate for high-risk patients for anesthesia (13). Nutritional status was assessed using the Modified Nutrition Alert Form (modified NAF) (14), and nutritional status was improved, as needed, to achieve target calorie intake. The energy requirement was $25-30 \mathrm{kcal} / \mathrm{kg} /$ day of total calories, and $1.5 \mathrm{~g} / \mathrm{kg} /$ day of protein. Nutrition supplement was given to moderately malnourished patients. Patients with severe malnutrition received nutritional support for at least 2 weeks prior to surgery. Enteral feeding tube insertion was indicated in patients who could not tolerate adequate oral intake (3).

During the perioperative period, body positioning and how to cough were taught to patients. Patients with gastric outlet obstruction underwent gastric lavage and decompression at least 3 days before surgery. Postoperative nausea and vomiting (PONV) risk was assessed, and prophylaxis would be prescribed in patients with 2 or more risk factors. On the day of surgery, patients were allowed to have a normal meal until 6 hours before surgery, and drink clear liquid until 3 hours before the operation. Preoperative oral carbohydrate loading with apple juice or 50 grams of glucose solution was administered to all non-diabetic patients at 3 to 4 hours before surgery. Maintenance intravenous fluid was administered in the morning to prevent dehydration. During the operation, compression stockings were used for thromboembolism prophylaxis. An air warming blanket was applied to prevent hypothermia. Patients received antimicrobial prophylaxis at least 30 minutes before skin incision. If the duration of the operation was longer than 4 hours or if the estimated blood loss was greater than $500 \mathrm{ml}$, an additional dose of antibiotic was administered. An epidural or intravenous patient-controlled analgesia (PCA) was given to provide adequate postoperative pain control. We avoided placement of a nasogastric tube and/or external drain whenever possible. Perioperative glycemic control was evaluated and maintained.

On postoperative day (POD) 1, patients were encouraged to walk and allowed to sip water. Patients started to drink liquid diet on POD 2, then allowed to 
ingest low residual soft diet on POD 3. All drains were removed as soon as possible. Patients could be discharged on POD 4 if they satisfied the discharge criteria.

Patients who underwent transthoracic esophagectomy routinely received epidural analgesia and intercostal drainage. Enteral feeding was started via a jejunostomy feeding tube if hemodynamic status was stable. Chest physical therapy was initiated as early as possible after surgery (11). After extubation, patients were allowed to sip water on POD 3. Patients started to drink a liquid diet on postoperative day 4 . Watersoluble contrast UGI study was performed after postoperative day 7 . If the results of that study showed patent anastomosis, low residual soft food intake was allowed. Patients could be discharged on postoperative day 8 if they met the discharge criteria $(6,11)$.

The discharge criteria included a normal body temperature, hemodynamic stability, adequate pain relief with oral analgesics, normal bowel movement, tolerance of soft diet, and normal white blood cell count. Our team contacted patients by telephone at 48-72 hours after discharge for clinical follow-up, then routine postoperative surveillance was performed. Patients were advised that they could contact us at any time if they had a problem.

\section{Data collection}

Clinicopathological characteristics were recorded, including age, gender, body mass index (BMI), ASA classification, comorbidities, preoperative diagnosis, tumor location, preoperative clinical and pathological staging, resection category, operative approach, and surgical procedure. Cancer of the esophagus, EGJ, and stomach were staged based on the $7^{\text {th }}$ edition American Joint Committee on Cancer (AJCC) staging system $(15,16)$.

The primary outcome of this study was the total length of hospital stay (LOS) compared between the ERAS group and the CC group. The secondary outcomes were the incidence of postoperative complications, the recovery of intestinal function, postoperative mortality, hospitalization cost, readmission rate, nutritional status, and biochemical data compared between groups. Major complications were defined as grade $3 a$ or higher complications within 30 days after surgery according to Clavien-Dindo classification (17). Hypothermia was defined as an intraoperative core body temperature below $36^{\circ} \mathrm{C}$. The definition of intraoperative hypotension was mean arterial pressure less than $65 \mathrm{mmHg}$. Inadequate pain control was defined as a maximum pain score of 7 or higher on a visual analog scale (VAS; score 0-10) two or more consecutive times within the first 72 hours postoperatively. Hospitalization cost included all operative and anesthetic equipment, the cost of pathology and laboratory tests, imaging, operating room surgical costs, medications, but excluded special food and physician fee.

\section{Statistical analysis}

All data were analyzed using Statistical Package for the Social Sciences (SPSS) version 21.0 (SPSS, Inc., Chicago, IL, USA). Continuous data were compared using Student's t-test, and reported as mean \pm standard deviation or median and interquartile range. Categorical variables were analyzed using chi-square test or Fisher's exact test, and the results are reported as number and percentage. A p-value of less than 0.05 was considered statistically significant for all tests.

\section{RESULTS}

\section{Patient characteristics}

A total of 88 patients who underwent UGI surgery were enrolled. Thirty-two patients were treated with ERAS protocol (ERAS group), and fifty-six patients received conventional care (CC group). There were no significant differences between groups relative to diagnosis and operation (fig. 1). Patient characteristics and baseline data showed no significant differences between groups, except for operative approach (table 1). The median (interquartile range [IQR]) duration of intraoperative hypotension and hypothermia in ERAS and CC patients were $5(0-20)$ vs. $5(0-23.7)$ minutes $(p=0.238)$, and $67.5(0-187.5)$ vs. $15.0(0-236.2)$ minutes $(p=0.964)$, respectively.

\section{Compliance with the ERAS protocol}

Education and counseling, smoking and alcohol cessation, anti-thrombotic prophylaxis, and avoidance of hypothermia was achieved in all ERAS patients, and adequate nutrition support was achieved in $93.8 \%$ of ERAS patients. Preoperative fasting and carbohydrate loading was ordered in $81.3 \%$. PONV prophylaxis and antimicrobial prophylaxis, including redose, were prescribed in $90.6 \%$. Intraoperative hypothermia occurred in $53.2 \%$, and intraoperative hypotension developed in $56.3 \%$. Rate of ambulation on POD 3 was $78.1 \%$, and inadequate pain control was observed in $15.6 \%$. Overall compliance with the ERAS protocol in this study was $86.9 \%$. 


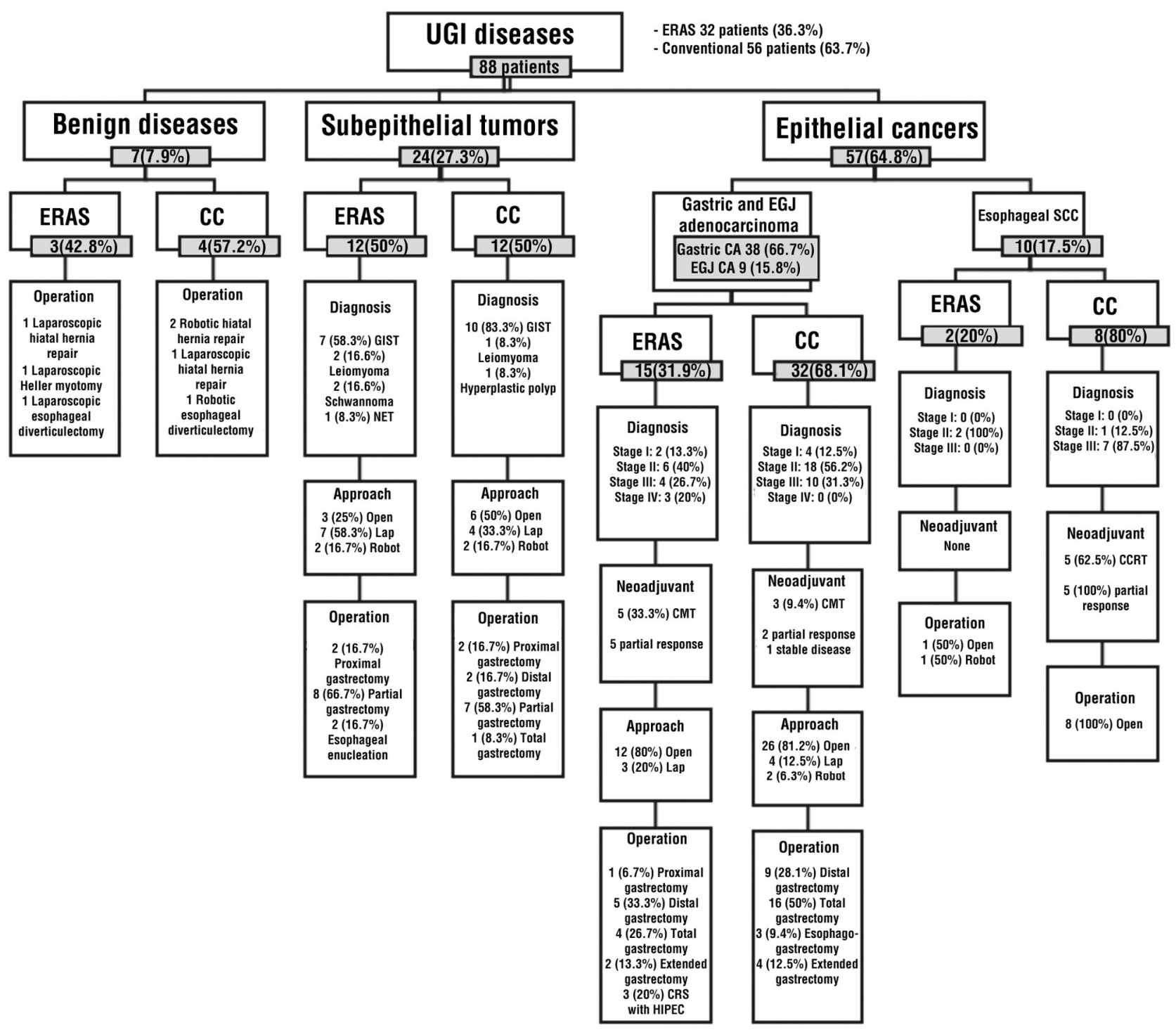

Figure 1 - Upper gastrointestinal diseases and operations

Abbreviations: CA, cancer; CC, conventional care; CCRT, concurrent chemoradiotherapy; CMT, chemotherapy; CRS with HIPEC, cytoreductive surgery with hyperthermic intraperitoneal chemotherapy; EGJ, esophagogastric junction; ERAS, Enhanced Recovery after Surgery; GIST, gastrointestinal stromal tumor; Lap, laparoscopic surgery; NET, neuroendocrine tumor; Open, open surgery; Robot, robotic surgery; SCC, squamous cell carcinoma; UGI, upper gastrointestinal

\section{Short-term outcomes after UGI surgery}

The median length of hospital stay (LOS) was significantly shorter in the ERAS group than in the CC group (5.5 vs. 12.5 days, $p<0.001$ ). Time to toleration of oral water, liquid diet, and soft diet was significantly earlier in ERAS than in CC (all $p<0.001$ ). ERAS patients also had significantly faster recovery of intestinal function. The mean time to first defecation was significantly shorter in ERAS than in CC (71.6 vs. 114.5 hours, $\mathrm{p}<0.001)$. Patients in the ERAS group also had earlier postoperative ambulation compared to their CC counterparts (43.8 vs. 84.6 hours, $\mathrm{p}=0.010$ ). ERAS patients had a significantly lower mean hospitalization cost than CC group patients $(197,140.0$ vs. $256,314.8$ Thai baht [THB], $\mathrm{p}=0.032$ ) (table 2).

\section{Complication after UGI surgery}

The overall postoperative complication rate was not significantly different between the ERAS and CC groups (31.2\% vs. $39.3 \%, p=0.497$ ). Regarding major complications, one (3.1\%) patient in the ERAS group developed pneumonia after transthoracic esophagectomy that 
Table 1 - Demographic and clinical characteristics compared between the ERAS and CC groups

\begin{tabular}{|c|c|c|c|}
\hline Characteristics & $\begin{array}{c}\text { ERAS group } \\
(\mathrm{n}=32)\end{array}$ & $\begin{array}{c}\text { CC group } \\
(n=56)\end{array}$ & p-value \\
\hline Age (years), mean $\pm S D$ & $58.9 \pm 15.6$ & $63.5 \pm 12.3$ & 0.134 \\
\hline Gender (male:female), $\mathrm{n}$ & $12: 20$ & $30: 26$ & 0.185 \\
\hline Body mass index $\left(\mathrm{kg} / \mathrm{m}^{2}\right)$, mean $\pm \mathrm{SD}$ & $23.1 \pm 3.7$ & $21.8 \pm 4.0$ & 0.125 \\
\hline $\begin{array}{l}\text { ASA classification, } n(\%) \\
\text { I-II } \\
\text { III-IV }\end{array}$ & $\begin{array}{c}24(75.0 \%) \\
8(25.0 \%)\end{array}$ & $\begin{array}{l}34(60.7 \%) \\
22(39.3 \%)\end{array}$ & 0.243 \\
\hline $\begin{array}{l}\text { Comorbidity, } \mathrm{n}(\%) \\
\text { Diabetes mellitus } \\
\text { Hypertension } \\
\text { Cerebrovascular disease } \\
\text { COPD } \\
\text { Coronary artery disease } \\
\text { Valvular heart disease } \\
\text { Arrhythmia } \\
\text { Chronic kidney disease* } \\
\text { Stage 1 } \\
\text { Stage 2-3 } \\
\text { Stage 4-5 }\end{array}$ & $\begin{array}{c}15(46.9 \%) \\
5(15.6 \%) \\
14(43.8 \%) \\
2(6.3 \%) \\
0(0.0 \%) \\
0(0.0 \%) \\
1(3.1 \%) \\
1(3.1 \%) \\
30(93.8 \%) \\
2(6.2 \%) \\
0(0.0 \%)\end{array}$ & $\begin{array}{c}32(57.1 \%) \\
11(19.6 \%) \\
23(41.1 \%) \\
5(8.9 \%) \\
1(1.8 \%) \\
6(10.7 \%) \\
0(0.0 \%) \\
5(8.9 \%) \\
\\
50(89.3 \%) \\
4(7.1 \%) \\
2(3.6 \%)\end{array}$ & $\begin{array}{l}0.382 \\
0.777 \\
0.826 \\
1.000 \\
1.000 \\
0.082 \\
0.364 \\
0.410 \\
1.000\end{array}$ \\
\hline $\begin{array}{l}\text { Operative approach, n (\%) } \\
\text { Open surgery } \\
\text { Laparoscopic surgery } \\
\text { Robotic surgery }\end{array}$ & $\begin{array}{c}16(50.0 \%) \\
13(40.6 \%) \\
3(9.4 \%)\end{array}$ & $\begin{array}{l}40(71.4 \%) \\
9(16.1 \%) \\
7(12.5 \%)\end{array}$ & 0.038 \\
\hline $\begin{array}{l}\text { Operation, } \mathrm{n}(\%) \\
\text { Procedure for benign disease } \\
\text { Esophageal surgery } \\
\text { Gastric surgery }\end{array}$ & $\begin{array}{c}3(9.4 \%) \\
4(12.5 \%) \\
25(78.1 \%)\end{array}$ & $\begin{array}{c}4(7.1 \%) \\
11(19.7 \%) \\
41(73.2 \%)\end{array}$ & 0.932 \\
\hline
\end{tabular}

A p-value $<0.05$ indicates statistical significance

*Chronic kidney disease stage 1, 2, 3, 4, and 5 are defined as kidney damage or GFR >90, 60-89, 30-59, 15-29, and <15 $\mathrm{ml} / \mathrm{min}$ per $1.73 \mathrm{~m} 2$, respectively

Abbreviations: ERAS, Enhanced Recovery after Surgery; CC, conventional care; SD, standard deviation; n, number; ASA, American Society of Anesthesiologists; CC, conventional care; COPD, chronic obstructive pulmonary disease; GFR, glomerular filtration rate

required prolonged endotracheal intubation for respiratory support. In the CC group, major complications occurred in 6 (10.7\%) patients. One patient had anastomotic bleeding after open total gastrectomy that required endoscopic evaluation. One patient had empyema thoracis after Ivor-Lewis esophagectomy that was treated by chest tube drainage. Three patients that underwent transthoracic esophagectomy suffered from respiratory complications that included aspiration pneumonia, pleural effusion, and pneumothorax requiring respiratory support and chest drainage. Another one patient suffered from respiratory failure and required re-endotracheal tube intubation after esophageal diverticulectomy.

Surgery-related complications were not significantly different between the ERAS and CC groups $(21.9 \%$ vs. $17.9 \%, p=0.780)$. In ERAS, 5 (15.6\%) patients had biochemical pancreatic fistula, and 1 (3.1\%) patient suffered from total splenic infarction after gastric surgery. Left true vocal cord paralysis was noted in 1 (3.1\%) patient after transthoracic esophagectomy.
All of the immediately aforementioned patients were successfully treated with non-operative treatment. In CC, $3(5.4 \%)$ patients had surgical site infection, 1 (1.8\%) patient had postoperative anemia requiring blood transfusion, 1 (1.8\%) patient had bowel ileus, $1(1.8 \%)$ patient had duodenal stump leakage, 1 (1.8\%) patient developed ascites, and 3 (5.4\%) patients had pancreatic fistula. For transthoracic esophagectomy, one (1.8\%) patient suffered from esophagogastric anastomosis leakage that required endoscopic esophageal stenting, and one (1.8\%) patient had left true vocal cord paralysis. The 28-day readmission rate was $0.0 \%$ in the ERAS group, but $3.6 \%$ ( 2 patients) in the CC group ( $p=0.532)$. One of those was readmitted due to anastomotic stricture that was treated with endoscopic esophageal stent placement. The other patient suffered from jejunostomy feeding tube dislodge that required jejunostomy feeding tube reinsertion. No mortality was reported in either group during the 30-day follow-up period. 
Table 2 - Postoperative short-term outcome data after UGI surgery compared between the ERAS and CC groups

\begin{tabular}{|c|c|c|c|}
\hline Outcome data & $\begin{array}{c}\text { ERAS group } \\
(\mathrm{n}=32)\end{array}$ & $\begin{array}{c}\text { CC group } \\
(n=56)\end{array}$ & p-value \\
\hline ICU admission (hours), median (IQR) & $0(0-10.1)$ & $0(0-22.2)$ & 0.670 \\
\hline ETT (hours), median (IQR) & $0(0-0)$ & $0(0-0)$ & 0.806 \\
\hline $\begin{array}{l}\text { Oral intake (POD), median (IQR) } \\
\text { Water } \\
\text { Liquid diet } \\
\text { Soft diet }\end{array}$ & $\begin{array}{l}1.0(1-1) \\
2.0(2-2) \\
3.0(3-3)\end{array}$ & $\begin{array}{c}7.0(3.2-8) \\
7.0(4.2-8.7) \\
8.0(6-10)\end{array}$ & $\begin{array}{l}<0.001 \\
<0.001 \\
<0.001\end{array}$ \\
\hline $\begin{array}{l}\text { Bowel movement (hours), mean } \pm S D \\
\text { First flatus } \\
\text { First defecation }\end{array}$ & $\begin{array}{l}51.8 \pm 25.3 \\
71.6 \pm 28.2\end{array}$ & $\begin{array}{c}67.4 \pm 32.9 \\
114.5 \pm 37.0\end{array}$ & $\begin{array}{c}0.055 \\
<0.001\end{array}$ \\
\hline Ambulation (hours), mean $\pm S D$ & $43.8 \pm 33.0$ & $84.6 \pm 70.2$ & 0.010 \\
\hline Inadequate pain control, $\mathrm{n}(\%)$ & $7(12.5 \%)$ & $5(15.6 \%)$ & 0.751 \\
\hline $\begin{array}{l}\text { Complication, n (\%) } \\
\text { No } \\
\text { Minor } \\
\text { Major }\end{array}$ & $\begin{array}{c}22(68.8 \%) \\
9(28.1 \%) \\
1(3.1 \%)\end{array}$ & $\begin{array}{l}34(60.7 \%) \\
16(28.6 \%) \\
6(10.7 \%)\end{array}$ & 0.497 \\
\hline $\begin{array}{l}\text { Complication, } \mathrm{n}(\%) \\
\text { Surgery-related } \\
\text { Non-surgery-related }\end{array}$ & $\begin{array}{l}7(21.9 \%) \\
4(12.5 \%)\end{array}$ & $\begin{array}{l}10(17.9 \%) \\
17(30.4 \%)\end{array}$ & $\begin{array}{l}0.780 \\
0.072\end{array}$ \\
\hline 28-day readmission, $\mathrm{n}(\%)$ & $0(0.0 \%)$ & $2(3.6 \%)$ & 0.532 \\
\hline In-hospital mortality, n (\%) & $0(0.0 \%)$ & $0(0.0 \%)$ & \\
\hline 30 -day postoperative mortality, $\mathrm{n}(\%)$ & $0(0.0 \%)$ & $0(0.0 \%)$ & \\
\hline POS (days), median (IQR) & $5.0(4-6)$ & $10.0(8.2-13)$ & $<0.001$ \\
\hline LOS (days), median (IQR) & $5.5(5-7)$ & $12.5(9.2-15.7)$ & $<0.001$ \\
\hline Hospitalization cost (THB), mean $\pm S D$ & $197,140 \pm 120,567$ & $256,315 \pm 123,214$ & 0.032 \\
\hline
\end{tabular}

A p-value $<0.05$ indicates statistical significance

Abbreviations: UGI, upper gastrointestinal; ERAS, Enhanced Recovery after Surgery; CC, conventional care;

ICU, intensive care unit; IQR, interquartile range; ETT, endotracheal tube; POD, postoperative day; SD, standard deviation;

n, number; POS, postoperative length of hospital stay; LOS, length of hospital stay; THB, Thai baht

\section{Inflammatory markers}

C-reactive protein (CRP) level (fig. 2), white blood cell (WBC) count, and absolute lymphocyte count (ALC) (fig. 3) were all comparable between groups during the preoperative period. In the early postoperative period, CRP and WBC levels in ERAS patients showed a nonsignificant trend toward being lower than those in CC; however, the ALC in the ERAS groups was higher than that in the CC group. The CRP level on POD 1 in ERAS was significantly lower than in CC (61.3 vs. $87.9 \mathrm{mg} / \mathrm{L}$, $p=0.027$ ). The point of maximum peak (on POD 3) was not significantly different between groups (101.2 vs. $126.9 \mathrm{mg} / \mathrm{L}, \mathrm{p}=0.202$ ).

Body weight (BW) and body mass index (BMI)

There was no significant difference between groups for preoperative body weight ( 58.3 vs. $56.6 \mathrm{~kg}$, $\mathrm{p}=0.522)$ or BMI (22.9 vs. $\left.21.7 \mathrm{~kg} / \mathrm{m}^{2}, \mathrm{p}=0.170\right)$. At 3 months after surgery, BMI in ERAS was significantly higher than in $\mathrm{CC}$ (21.0 vs. $16.8, \mathrm{p}=0.035)$. The average BMI reduction was $7.4 \%$ vs. $11.1 \%(p=0.126)$ at 1 month, and $8.3 \%$ vs. $22.6 \%$ ( $p=0.275)$ at 3 months postoperatively in ERAS and CC, respectively. Postoperative BMI recovery was observed within 3 months in ERAS patients, and within 6 months in CC patients.

\section{Subgroup of benign diseases}

The characteristics of patients who underwent benign UGI procedures showed no statistically significant differences between the ERAS and CC groups. There was also no significant difference for mean LOS between ERAS and CC (4.7 vs. 7.0 days, $\mathrm{p}=0.211)$. ERAS had a significantly lower hospitalization cost than CC ( 97,528 vs. $350,513 \mathrm{THB}, \mathrm{p}=0.044)$. There were no differences between groups for postoperative recovery or complications. There were no postoperative complications in ERAS, but one (25.0\%) major complication was observed in CC. No 28-day readmission or 30-day mortality was reported. 

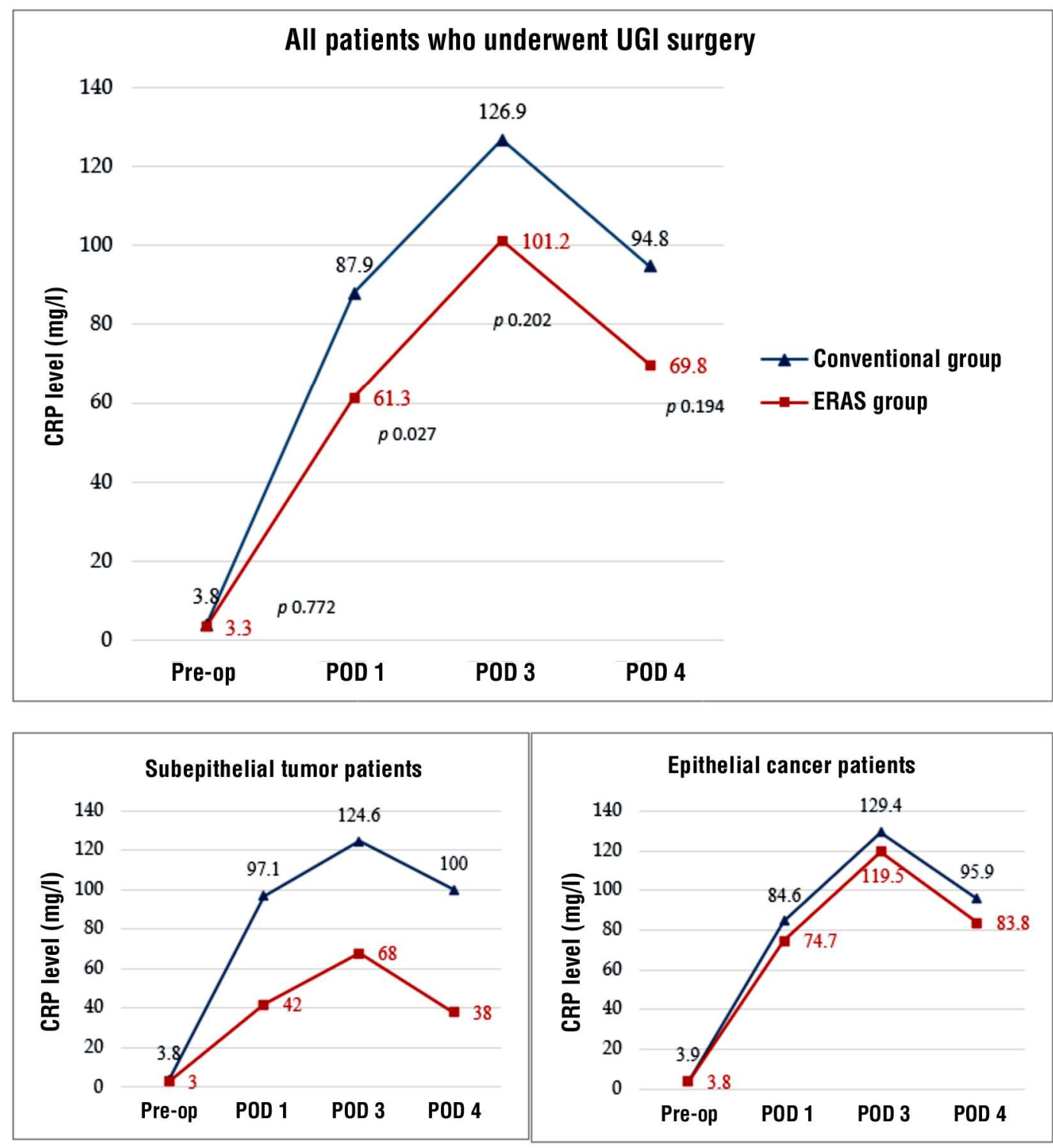

Figure 2 - Mean postoperative C-reactive protein (CRP) levels compared between Enhanced Recovery after Surgery (ERAS) and conventional care patients among all upper gastrointestinal (UGI) surgery patients, in patients with subepithelial tumors, and in patients with epithelial cancer (Abbreviations: pre-op, preoperative; POD, postoperative day)

\section{Subgroup of subepithelial tumors}

ERAS patients had a significantly shorter median time to toleration of oral soft diet (3 vs. 5.5 days, $\mathrm{p}<0.001)$, shorter median time to first defecation (65.8 vs. 110.6 hours, $p=0.032$ ), and shorter LOS ( 5 vs. 7 days, $\mathrm{p}<0.001)$ than CC patients. The hospitalization cost of ERAS patients was lower than in CC, but the difference between groups was not statistically significant (163,777 vs. 209,506 THB, $p=0.351)$. There was no postoperative complication in ERAS patients. Among CC patients, no major complication occurred, but minor complication occurred in 3 patients $(0.0 \%$ vs. $25.0 \%$, $\mathrm{p}=0.217$ ). No 28 -day readmission or 30 -day mortality was observed.

\section{Subgroup of epithelial tumors}

ERAS and CC patients had comparable demographic data and tumor characteristics (table 3). Adeno-carcinoma was the most common histologic type, comprising $88.2 \%$ in ERAS and $80 \%$ in CC. Tumor-free resection margin (RO resection) was achieved in all ERAS patients, but in only $85 \%$ of CC patients. There were no significant differences in the mean number of harvested lymph nodes for adeno- 

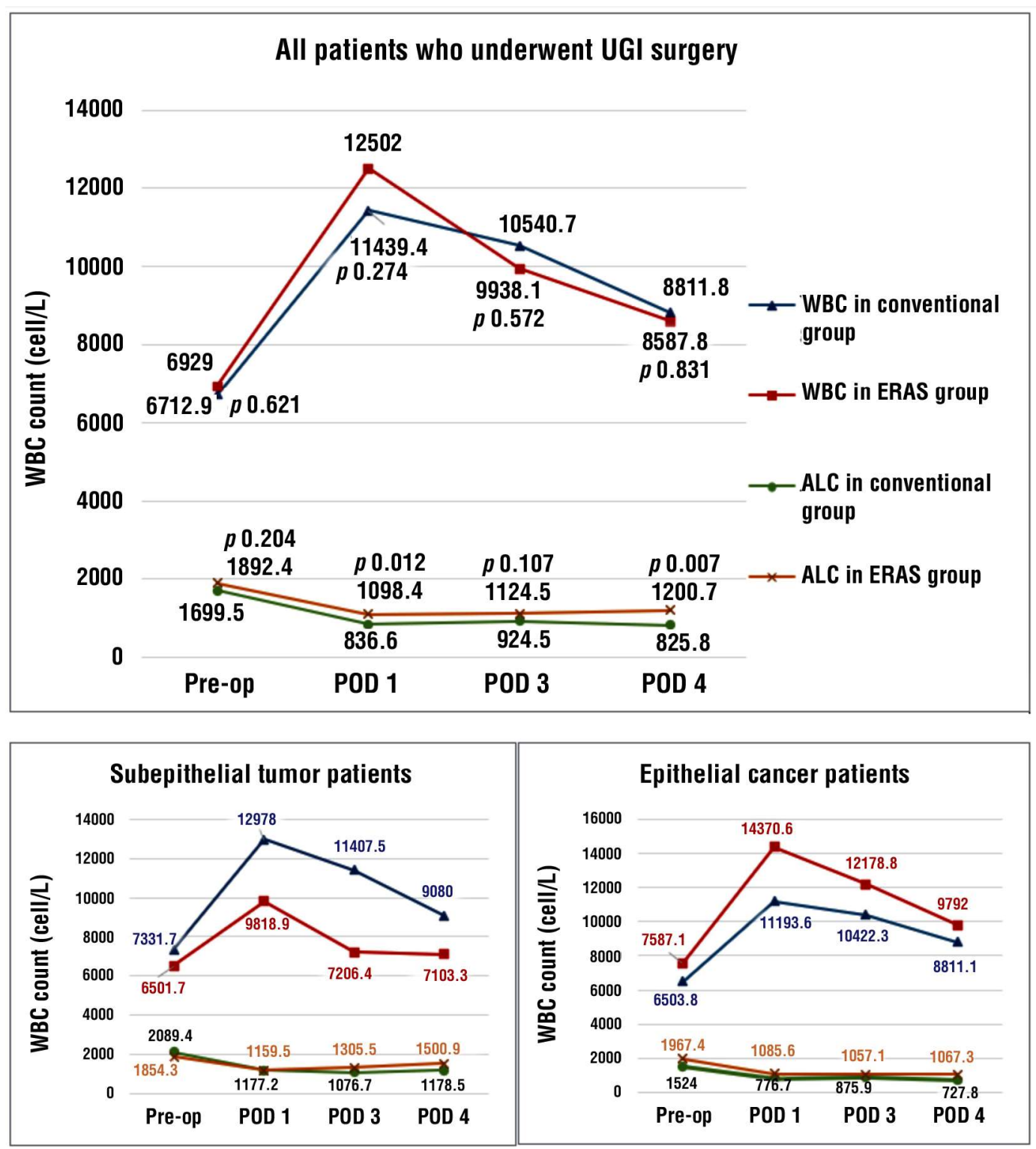

Figure 3 - Mean postoperative white blood cell (WBC) and absolute lymphocyte count (ALC) levels compared between Enhanced Recovery after Surgery (ERAS) and conventional care patients among all upper gastrointestinal (UGI) surgery patients, in patients with subepithelial tumors, and in patients with epithelial cancer (Abbreviations: POD, postoperative day; Pre-op, preoperative day).

carcinoma and squamous cell carcinoma between ERAS and CC $(55.0 \pm 24.2$ vs. $37.3 \pm 15.9$ nodes, $\mathrm{p}=0.140$, and $82.0 \pm 56.5$ vs. $38.1 \pm 21.4$ nodes, $\mathrm{p}=0.140$ respectively). ERAS had significantly shorter time to toleration of oral diet (all $p<0.001$ ) and shorter LOS than in CC (7.5 vs. 15.6 days, $p<0.001)$. The incidence of major postoperative complication was not significantly different between ERAS and CC (5.9\% vs. $12.5 \%, p=0.395)$. No postoperative mortality was observed in either group. Hospitalization cost was not significantly different between groups (table 4).

\section{Adenocarcinoma of stomach and esophago- gastric junction}

Mean LOS was significantly shorter in ERAS than in CC (6.9 vs. 14.9 days, $p<0.001$ ). Time to toleration of oral intake was significantly shorter in ERAS than in CC (all $p<0.001$ ). In ERAS patients, the mean time to toleration of oral water, liquid diet, and soft diet was POD 1.4, 2.4, and 3.9, respectively. Among CC, the average time to toleration of oral water, liquid diet, and soft diet was POD 6.8, 7.6, and 8.8, respectively. Mean time to first defecation was significantly shorter in the ERAS group (73.0 vs. 110.1 hours, $p<0.001$ ). Patients in 
Table 3 - Tumor characteristics, surgical data, and pathological data compared between the ERAS and CC groups

\begin{tabular}{|c|c|c|c|}
\hline Data & ERAS group $(n=17)$ & CC group $(n=40)$ & $\mathrm{p}$-value \\
\hline$\overline{\text { Age (years), mean } \pm S D}$ & $56.4 \pm 15.4$ & $64.0 \pm 13.2$ & 0.064 \\
\hline Gender, $n$ (male:female) & $7: 10$ & $21: 19$ & 0.565 \\
\hline $\begin{array}{l}\text { Tumor location, } \mathrm{n}(\%) \\
\text { Esophagus } \\
\text { Esophagogastric junction } \\
\text { Stomach }\end{array}$ & $\begin{array}{c}2(11.8 \%) \\
4(23.5 \%) \\
11(64.7 \%)\end{array}$ & $\begin{array}{c}8(20.0 \%) \\
5(12.5 \%) \\
27(67.5 \%)\end{array}$ & 0.304 \\
\hline $\begin{array}{l}\text { Histopathology, } \mathrm{n}(\%) \\
\text { Squamous cell carcinoma } \\
\text { Adenocarcinoma }\end{array}$ & $\begin{array}{c}2(11.8 \%) \\
15(88.2 \%)\end{array}$ & $\begin{array}{c}8(20.0 \%) \\
32(80.0 \%)\end{array}$ & 0.706 \\
\hline $\begin{array}{l}\text { Squamous cell carcinoma } \\
\text { Operation, } \mathrm{n}(\%) \\
\text { Total esophagectomy } \\
\text { pT stage, } \mathrm{n}(\%) \\
\text { T 0-2 } \\
\text { T } 3-4 \\
\text { pN stage, n (\%) } \\
\text { N0 } \\
\text { N1-3 } \\
\text { Resection category, n (\%) } \\
\text { R0 } \\
\text { R1 } \\
\text { R2 }\end{array}$ & $\begin{array}{l}2(100 \%) \\
1(50.0 \%) \\
1(50.0 \%) \\
2(100 \%) \\
0(0.0 \%) \\
2(100 \%) \\
0(0.0 \%) \\
0(0.0 \%)\end{array}$ & $\begin{array}{c}8(100 \%) \\
2(25.0 \%) \\
6(75.0 \%) \\
4(50.0 \%) \\
4(50.0 \%) \\
7(87.5 \%) \\
1(12.5 \%) \\
0(0.0 \%)\end{array}$ & $\begin{array}{l}1.000 \\
1.000 \\
0.467\end{array}$ \\
\hline $\begin{array}{l}\text { Adenocarcinoma } \\
\text { Operation, n (\%) } \\
\text { Proximal gastrectomy } \\
\text { Distal gastrectomy } \\
\text { Total gastrectomy } \\
\text { Extended gastrectomy } \\
\text { Ivor-Lewis esophagectomy } \\
\text { CRS with HIPEC } \\
\text { pT stage, n (\%) } \\
\text { T 0-2 } \\
\text { T 3-4 } \\
\text { pN stage, n (\%) } \\
\text { N0 } \\
\text { N1-3 } \\
\text { Resection category, n (\%) } \\
\text { R0 } \\
\text { R1 } \\
\text { R2 }\end{array}$ & $\begin{array}{c}1(6.7 \%) \\
5(33.3 \%) \\
4(26.7 \%) \\
2(13.3 \%) \\
0(0.0 \%) \\
3(20.0 \%) \\
2(13.3 \%) \\
13(86.7 \%) \\
4(26.7 \%) \\
11(73.3 \%)\end{array}$ & $\begin{array}{c}0(0.0 \%) \\
9(28.1 \%) \\
16(50.0 \%) \\
4(12.5 \%) \\
3(9.4 \%) \\
0(0.0 \%) \\
9(28.1 \%) \\
23(71.9 \%) \\
7(21.9 \%) \\
25(78.1 \%)\end{array}$ & 0.461 \\
\hline
\end{tabular}

A p-value $<0.05$ indicates statistical significance

Abbreviations: ERAS, Enhanced Recovery after Surgery; CC, conventional care; SD, standard deviation; $n$, number; pT, pathological primary tumor stage; pN, pathological lymph node stage; T, tumor; N, node; R, resection category; CRS, cytoreductive surgery; HIPEC, hyperthermic intraperitoneal chemotherapy

the ERAS group demonstrated non-significantly earlier postoperative ambulation ( 46 vs. 71.3 hours, $p=0.086$ ). Postoperative major complications were non-significantly different between ERAS and CC $(0.0 \%$ vs. $6.3 \%$, $\mathrm{p}=0.755)$. There was also no significant difference in hospitalization cost between ERAS and CC patients (214,946 vs. $243,321 \mathrm{THB}, \mathrm{p}=0.511)$.

\section{Squamous cell carcinoma of esophagus}

All esophageal squamous cell carcinoma patients underwent esophagectomy with three-field lymphadenectomy. Mean LOS was not significantly different between ERAS and CC (12.0 vs. 18.2 days, $p=0.252$ ). Mean time to toleration of oral water (4.0 vs. 10.2 days, $p=0.303$ ), liquid diet ( 6.5 vs. 11.5 days, $p=0.402$ ), and soft diet (9.0 vs. $13.8, p=0.366$ ) was not significantly different between ERAS and CC patients. There was also no significant difference between ERAS and CC for time to postoperative ambulation (103.5 vs. 172 hours, $\mathrm{p}=0.538)$ or major complications $(50.0 \%$ vs. $37.5 \%$, $\mathrm{p}=0.467$ ). Pulmonary complication was the most common non-surgery-related complication in both ERAS and CC patients ( $6.7 \%$ vs. $15.6 \%, p=0.725)$. No postoperative mortality was observed in either group. There was no significant difference in hospitalization 
Table 4 - Postoperative short-term outcomes of epithelial tumors compared between the ERAS and CC groups

\begin{tabular}{|c|c|c|c|}
\hline Outcomes & ERAS group $(n=17)$ & CC group $(n=40)$ & p-value \\
\hline ICU admission (hours), median (IQR) & $0(0-32)$ & $0(0-46.7)$ & 0.840 \\
\hline ETT (hours), median (IQR) & $0(0-16)$ & $0(0-3)$ & 0.560 \\
\hline $\begin{array}{l}\text { Oral intake (POD), median (IQR) } \\
\text { Water } \\
\text { Liquid } \\
\text { Soft diet }\end{array}$ & $\begin{array}{l}1.0(1-2.5) \\
2.0(2-4) \\
3.0(3-6)\end{array}$ & $\begin{array}{c}7.0(6-8) \\
8.0(6.2-9) \\
9.0(7.2-11)\end{array}$ & $\begin{array}{l}<0.001 \\
<0.001 \\
<0.001\end{array}$ \\
\hline $\begin{array}{l}\text { Bowel movement (hours), mean } \pm \text { SD } \\
\quad \text { First flatus } \\
\text { First defecation }\end{array}$ & $\begin{array}{l}60.4 \pm 25.7 \\
77.4 \pm 33.4\end{array}$ & $\begin{array}{c}69.9 \pm 33.4 \\
117.6 \pm 34.7\end{array}$ & $\begin{array}{l}0.349 \\
0.001\end{array}$ \\
\hline Ambulation (hours), mean $\pm S D$ & $49.5 \pm 36.1$ & $93.7 \pm 73.9$ & 0.036 \\
\hline Inadequate pain control, n (\%) & $6(15.0 \%)$ & $4(23.5 \%)$ & 0.464 \\
\hline $\begin{array}{l}\text { Complication, n (\%) } \\
\text { No } \\
\text { Minor } \\
\text { Major }\end{array}$ & $\begin{array}{c}7(41.2 \%) \\
9(52.9 \%) \\
1(5.9 \%)\end{array}$ & $\begin{array}{l}22(55.0 \%) \\
13(32.5 \%) \\
5(12.5 \%)\end{array}$ & 0.395 \\
\hline $\begin{array}{l}\text { Complication, n (\%) } \\
\quad \text { Surgery-related } \\
\text { Non-surgery-related }\end{array}$ & $\begin{array}{l}7(41.2 \%) \\
4(23.5 \%)\end{array}$ & $\begin{array}{l}10(25.0 \%) \\
13(32.5 \%)\end{array}$ & $\begin{array}{l}0.342 \\
0.752\end{array}$ \\
\hline 28-day readmission, $\mathrm{n}(\%)$ & $0(0.0 \%)$ & $2(5.0 \%)$ & \\
\hline In-hospital mortality, $\mathrm{n}(\%)$ & $0(0.0 \%)$ & $0(0.0 \%)$ & \\
\hline 30-day postoperative mortality, $\mathrm{n}(\%)$ & $0(0.0 \%)$ & $0(0.0 \%)$ & \\
\hline POS (days), median (IQR) & $6.0(5-8.5)$ & $11.0(9-14)$ & $<0.001$ \\
\hline LOS (days), mean $\pm S D$ & $7.5 \pm 2.3$ & $15.6 \pm 7.4$ & $<0.001$ \\
\hline Hospitalization cost (THB), mean \pm SD & $238,270 \pm 103,150$ & $260,938 \pm 124,093$ & 0.511 \\
\hline
\end{tabular}

cost between the ERAS and CC groups $(413,199$ vs. 331,403 THB, $p=0.511)$.

\section{DISCUSSIONS}

The benefit of ERAS protocol in UGI surgery, including hepatobiliary and pancreatic surgery, gastric surgery, and esophageal surgery, has been reported in several studies $(18,19)$. Recently, the ERAS Society published evidence-based guidelines for enhanced recovery after gastrectomy and esophagectomy (10, 11). A higher level of compliance with the ERAS protocol was reported to be associated with improved treatment outcomes (20). In the present study, the overall rates of both preoperative and postoperative ERAS protocol compliance were high. However, we did encounter some difficulty preventing intraoperative hypotension and hypothermia. Most previous studies (21) focused on UGI cancer, so there is a relative scarcity of data specific to subgroup analysis in benign diseases. The present study reported the results of the ERAS protocol in various UGI diseases. Even though the numbers of patients were relatively low, we also performed sub- group analysis that compared among benign diseases, subepithelial tumors, and malignant tumors.

Previous study reported the benefit of ERAS protocol for reducing hospital LOS (19). Our study found the LOS in ERAS to be significantly shorter than in CC. However, the LOS in benign diseases was not significantly different between ERAS and CC. This might be due to the very low number of patients, less anastomoses, and more non-complex procedures. Subgroup analysis of subepithelial and epithelial tumors revealed a significant difference in LOS between groups. In patients with subepithelial tumors, the LOS in the ERAS group was 5 days, which is shorter than the previously reported range of 5.4-9.8 days $(19,22)$. A meta-analysis found the ERAS protocol in gastric cancer to be associated with a significant reduction in LOS (23). Mean LOS was reported to range from 5.4 to 14.1 days (19). Our study found a mean LOS after radical gastrectomy of 6.9 days, which is comparable to that previous study. The number of studies that evaluated the ERAS protocol in esophageal surgery is relatively few. A recent meta-analysis of the ERAS protocol in esophageal cancer (24) showed ERAS to be associated 
with a significant reduction of LOS. In the present study, the number of esophageal cancer patients was low; however, the mean LOS in ERAS was shorter than in CC ( $p>0.05$ ). There were no cases of readmission among ERAS patients in this study.

Reducing the duration of fasting, early enteral feeding, and early ambulation enhanced the recovery of intestinal function (25). Nutrition support has been shown to improve patient recovery. Early mobilization also decreased complications, such as thromboembolic events and pulmonary complications (26). This study demonstrated that the ERAS protocol contributed to a significantly earlier time to toleration of oral diet and recovery of intestinal function in subepithelial and epithelial tumor patients. Major concerns after esophageal and gastric resection are anastomotic healing and leakage. Prolonged postoperative oral diet restriction has traditionally been ordered in conventional care patients. However, our data showed ERAS to be associated with early enteral feeding that resulted in good oral diet tolerability and bowel movement. No incidence of aspiration pneumonia, bowel ileus, or anastomotic leakage was observed. There were 3 extended gastrectomy with cytoreductive surgery with hyperthermic intraperitoneal chemotherapy (CRS with HIPEC), which is known to be an extensive high-risk procedure, in the ERAS group; however, all of those patients recovered well without postoperative ileus.

A recent meta-analysis in UGI surgery reported no significant difference in complication rates between ERAS and CC (21). The incidence of postoperative major and surgery-related complications was not significantly different between groups in this study. ERAS patients had some minor complications and a very low incidence of major complication. There was only one major complication, which was postoperative pneumonia after transthoracic esophagectomy with 3-field lymphadenectomy. Transthoracic esophagectomy for esophageal squamous cell carcinoma is one of the most invasive surgeries. Most of the published research (27) reported on esophageal cancer patients who underwent 2-field lymphadenectomy; however, 3-field lymphadenectomy is the standard of surgical care at our center. Two systematic reviews $(27,28)$ reported the 30-day mortality rate after esophagectomy to be $1.2-11.1 \%$. Interestingly, there was no postoperative mortality after esophagectomy or gastrectomy in this study.

The level of serum CRP has been proposed as a postsurgical marker to evaluate surgical stress response (29). A recent meta-analysis demonstrated that ERAS was associated with reduced serum inflammatory mediators (30). Our study found the preoperative CRP levels to be comparable between groups; however, postoperative CRP level showed a non-significant trend toward being lower in ERAS patients than in CC patients. The observed lower CRP level in ERAS might be associated with lower inflammatory response and less postoperative complications.

Reduction in BMI was non-significantly lower in ERAS than in CC. BMI recovery occurred within 3 months after surgery in the ERAS group, whereas BMI recovery took 6 months in the $\mathrm{CC}$ group. This difference between groups may be due to enhanced preoperative nutritional management, early postoperative enteral feeding, and faster recovery in ERAS patients compared to $\mathrm{CC}$ patients. The shortened recovery period observed in ERAS patients provided additional important benefits, especially in patients who were going to receive adjuvant systemic therapy.

Among all patients in this study, the hospitalization cost of UGI surgery was significantly lower in the ERAS group than in the CC group. Moreover, in subgroup analysis of benign diseases, ERAS also had a significantly lower hospitalization cost than CC. This may be explained by the higher rate of more expensive robotic surgery in the CC group. A trend towards lower hospitalization cost was observed in subepithelial and epithelial tumors; however, no statistically significant difference was observed.

This study has some mentionable limitations. First, our study population was small, and this may have limited the statistical power of our study to identify all significant differences and associations. Second, although our study was prospective in nature, it did not have a randomized controlled design. Third and last, there was variability in the surgical procedures used to treat patients enrolled in this study. However, this can be explained by the different types of UGI disease that were included.

\section{CONCLUSIONS}

The results of this prospective study demonstrated the efficacy and safety of the ERAS protocol compared to conventional care in patients who underwent UGI surgery at our super-tertiary referral center. ERAS was associated with reduction in LOS, time to toleration of oral soft food intake, time to intestinal recovery, time to ambulation, and hospitalization cost. Preoperative nutrition support and early postoperative enteral feeding among ERAS patients resulted in faster BMI recovery. ERAS patients also had lower postoperative CRP levels, which indicated lower inflammatory 
response. Application of the ERAS protocol in UGI surgery was shown to be feasible and safe as evidenced by no increase in postoperative morbidity or mortality.

\section{Acknowledgements}

The authors gratefully acknowledge the patients that generously agreed to participate in this study; Dr. Saowalak Hunnangkul for her assistance with statistical analysis; and, Miss Nongnard Thamnongdee for her assistance with data collection.

\section{Ethical approval}

This study was approved by the Institutional Review Board (IRB) of the Faculty of Medicine Siriraj Hospital, Mahidol University (certificate of approval no. Si 119/ 2019), and all patients provided written informed consent to participate.

\section{Conflict of interest}

All authors declare no personal or professional conflicts of interest, and no financial support from the companies that produce and/or distribute the drugs, devices, or materials described in this report.

\section{Funding disclosure}

This was an unfunded study.

\section{REFERENCES}

1. Tan SJ, Zhou F, Chen QY, Lin ZL, Li N. Systemic review of the safety and efficacy of fast-track surgery combined with laparoscopy in radical gastrectomy for gastric cancer. Zhonghua Wei Chang Wai Ke Za Zhi. 2013;16(10):974-80.

2. Kehlet $\mathrm{H}$. Multimodal approach to control postoperative pathophysiology and rehabilitation. Br J Anaesth. 1997;78(5):606-17.

3. Weimann A, Braga M, Carli F, Higashiguchi T, Hubner M, Klek S, et al. ESPEN guideline: Clinical nutrition in surgery. Clin Nutr. 2017 36(3):623-50.

4. Awad S, Varadhan KK, Ljungqvist O, Lobo DN. A meta-analysis of randomised controlled trials on preoperative oral carbohydrate treatment in elective surgery. Clin Nutr. 2013:32(1):34-44.

5. Apfel CC, Kranke P, Eberhart LH, Roos A, Roewer N. Comparison of predictive models for postoperative nausea and vomiting. $\mathrm{Br} J$ Anaesth. 2002;88(2):234-40.

6. Rubinkiewicz M, Witowski J, Su M, Major P, Pedziwiatr M. Enhanced recovery after surgery (ERAS) programs for esophagectomy. J Thorac Dis. 2019;11(Suppl 5):S685-S91.

7. Liu F, Wang W, Wang C, Peng X. Enhanced recovery after surgery (ERAS) programs for esophagectomy protocol for a systematic review and meta-analysis. Medicine (Baltimore). 2018;97(8):e0016.

8. Wang LH, Zhu RF, Gao C, Wang SL, Shen LZ. Application of enhanced recovery after gastric cancer surgery: An updated metaanalysis. World J Gastroenterol. 2018;24(14):1562-78.

9. Pearsall EA, Meghii Z, Pitzul KB, Aarts MA, McKenzie M, McLeod $\mathrm{RS}$, et al. A qualitative study to understand the barriers and enablers in implementing an enhanced recovery after surgery program. Ann Surg. 2015;261(1):92-6.

10. Mortensen K, Nilsson M, Slim K, Schafer M, Mariette C, Braga M, et al. Consensus guidelines for enhanced recovery after gastrectomy Enhanced Recovery After Surgery (ERAS(R)) Society recommenda- tions. Br J Surg. 2014:101(10):1209-29.

11. Low DE, Allum W, De Manzoni G, Ferri L, Immanuel A, Kuppusamy $\mathrm{M}$, et al. Guidelines for Perioperative Care in Esophagectomy: Enhanced Recovery After Surgery [ERAS(R)] Society Recommendations. World J Surg. 2019;43(2):299-330.

12. Parakonthun T, Parichardsombat N, Paredes HSR, Phalanusittheph C, Taweerutchana V, Trakarnsanga A, et al. Significance of microscopic residual tumor in adenocarcinoma of stomach and esophagogastric junction after gastrectomy with D2 lymphadenectomy. Siriraj Med J. 2018;70(2):95-102.

13. Parakonthun T, Nampoolsuksan C, Swangsri J, Yiengpruksawan A, Methasate A. Retrospective Analysis of the Outcomes in Elderly Patients with Adenocarcinoma of the Stomach and Esophagogastric Junction Following Three Different Treatments. Siriraj Med J. 2019; 71(6):449-57.

14. Komindrg S, Tangsermwong T, Janepanish P. Simplified malnutrition tool for Thai patients. Asia Pac J Clin Nutr. 2013;22(4):516-21.

15. Washington K. 7th edition of the AJCC cancer staging manual: stomach. Ann Surg Oncol. 2010;17(12):3077-9.

16. Rice TW, Blackstone EH, Rusch WW. 7th edition of the AJCC Cancer Staging Manual: esophagus and esophagogastric junction. Ann Surg Oncol. 2010;17(7):1721-4.

17. Dindo D, Demartines N, Clavien PA. Classification of surgical complications: a new proposal with evaluation in a cohort of 6336 patients and results of a survey. Ann Surg. 2004;240(2):205-13.

18. Dorcaratto D, Grande L, Pera M. Enhanced recovery in gastrointestinal surgery: upper gastrointestinal surgery. Dig Surg. 2013;30(1):70-8.

19. Huang ZD, Gu HY, Zhu J, Luo J, Shen XF, Deng QF, et al. The application of enhanced recovery after surgery for upper gastrointestinal surgery: Meta-analysis. BMC Surg. 2020;20(1):3.

20. Gustafsson UO, Oppelstrup $H$, Thorell A, Nygren J, Ljungqvist 0. Adherence to the ERAS protocol is Associated with 5-Year Survival After Colorectal Cancer Surgery: A Retrospective Cohort Study. World J Surg. 2016:40(7):1741-7.

21. Siotos C, Stergios K, Naska A, Frountzas M, Pergialiotis V, Perrea $D N$, et al. The impact of fast track protocols in upper gastrointestinal surgery: A meta-analysis of observational studies. Surgeon. 2018; 16(3):183-92.

22. Fujikuni N, Tanabe K, Tokumoto N, Suzuki T, Hattori M, Misumi T, et al. Enhanced recovery program is safe and improves postoperative insulin resistance in gastrectomy. World J Gastrointest Surg. 2016;8(5):382-8.

23. Li YJ, Huo TT, Xing J, An JZ, Han ZY, Liu XN, et al. Meta-analysis of efficacy and safety of fast-track surgery in gastrectomy for gastric cancer. World J Surg. 2014;38(12):3142-51.

24. Pisarska M, Malczak P, Major P, Wysocki M, Budzynski A, Pedziwiatr $M$. Enhanced recovery after surgery protocol in oesophageal cancer surgery: Systematic review and meta-analysis. PLoS One. 2017;12(3):e0174382.

25. Chen S, Zou Z, Chen F, Huang Z, Li G. A meta-analysis of fast track surgery for patients with gastric cancer undergoing gastrectomy. Ann R Coll Surg Engl. 2015;97(1):3-10.

26. Zang K, Chen B, Wang M, Chen D, Hui L, Guo S, et al. The effect of early mobilization in critically ill patients: A meta-analysis. Nurs Crit Care. 2019

27. Mamidanna R, Bottle A, Aylin P, Faiz O, Hanna GB. Short-term outcomes following open versus minimally invasive esophagectomy for cancer in England: a population-based national study. Ann Surg. 2012;255(2):197-203

28. Blencowe NS, Strong S, McNair AG, Brookes ST, Crosby T, Griffin SM, et al. Reporting of short-term clinical outcomes after esophagectomy: a systematic review. Ann Surg. 2012;255(4):65866.

29. Miller RJ, Sutherland AG, Hutchison JD, Alexander DA. C-reactive protein and interleukin 6 receptor in post-traumatic stress disorder: a pilot study. Cytokine. 2001;13(4):253-5.

30. Moore FA, Feliciano DV, Andrassy RJ, McArdle AH, Booth FV, Morgenstein-Wagner TB, et al. Early enteral feeding, compared with parenteral, reduces postoperative septic complications. The results of a meta-analysis. Ann Surg. 1992;216(2):172-83. 